\title{
A MiCROMACHINED STAINLESS STEEL CUFF FOR ELECTROMAGNETIC MEASUREMENT OF FLOW IN BLOOD VESSELS
}

\author{
Kenichi Takahata* and Yogesh B. Gianchandani \\ Department of Electrical Engineering and Computer Science, University of Michigan, Ann Arbor
}

\begin{abstract}
This paper reports a micromachined cuff that senses blood flow velocity inside a blood vessel. Utilizing two electrically isolated electrodes in the cuff, the sensor produces a voltage proportional to the flow rate in the presence of a magnetic flux, which is to be externally read out by an implanted circuit chip. The cuff structure is fabricated from planar stainless steel foil using batch-compatible micro-electro-discharge machining and subsequently plastically reshaped into a ring by inflating a cylindrical balloon inside a blood vessel. The cuff has a pair of electrodes which are separated from each other by insulating links. A dielectric layer coats all surfaces except the electrodes. Devices deployed inside 3-mm i.d. silicone tubes demonstrate linear and symmetric responses of 3.1-4.3 V per cm/sec over $180 \mathrm{~cm} / \mathrm{sec}$ with fields of about $0.25 \mathrm{~T}$ created by a local permanent magnet of $25 \times 25 \times 9 \mathrm{~mm}^{3}$. Output dependences on the orientation of electrodes, magnetic field, and degree of stenosis (blockage inside a blood vessel) are also theoretically evaluated.
\end{abstract}

\section{INTRODUCTION}

Chronic measurement of blood flow is important for longterm monitoring of vascular diseases, including coronary artery disease, which is commonly treated by a medical procedure called balloon angioplasty with stent implantation. However, re-closures often occur due to recoil of the blood vessels or further plaque deposition. Wireless monitoring of blood flow can provide advance notice of such failures. Detection mechanisms proposed in the past for implantable sensors include thermal resistor [1], blood conductivity [1] and differential pressure measurement using capacitive diaphragms [2]. Electromagnetic detection [3] is another promising candidate since it has several potential advantages over each of these options, which include linear relationship between the output and flow, less output dependence on cross-sectional flow profile, and elimination of the sensing diaphragms, which can potentially improve reliability and lifetime. Electromagnetic flow sensors typically have two electrodes located on inner walls of the fluid channel. In the presence of a magnetic field, a voltage proportional to the flow velocity is developed between electrodes. The principle has been demonstrated in micro domain as well [4]. Two of the possible configurations for micromachined blood flow sensors are illustrated in Fig. 1: (A) a $\mathrm{C}$-shaped cuff with penetrating electrodes is wrapped around and a blood vessel, and (B) a circular cuff is located inside a blood vessel. In this effort, the latter approach is explored. The voltage is intended to be read out and transmitted by a separate device.

\section{DESIGN \& FABRICATION}

When all three orientations of blood flow, magnetic field, and electrical sense axis are perpendicular each other, the induced voltage $E$ is maximized and given by:

$$
E_{\max } D \cdot B \cdot v
$$

where $D$ is the diameter of the flow channel, $B$ is the magnetic flux density and $v$ is the cross-sectional average velocity of the flow. This linear equation assumes that (a) the magnetic field is spatially uniform, (b) the flow velocity profile is axially symmetric, and (c) the fluid is electrically conductive. The condition (b) is valid in typical arterial vessels and also downstream of a narrowed site if the location is reasonably away from the blockage. It is worth noting that despite the condition (c), the output voltage is independent of conductivity over a wide range [5].

The cuff presents challenges in both form and material. In the recent past, we have reported a fabrication technique for cardiac stents that are machined from planar steel foil by batch-compatible micro-electro-discharge machining ( EDM) [6] and plastically reshaped into a tubular shape by a standard balloon angioplasty procedure [7]. The completed structure did not use any bonded or hinged joints, and demonstrated excellent mechanical strength. The balloon expansion technique is suitable for tailoring the final diameter of the cuff to actual inner diameter of an artery at the location of the implant. The fabrication process of the stent is also useful for the cuff because it provides a planar approach to the micromachining of stainless steel, which is a suitable structural material. However, it does not accommodate insulating segments that will permit electrical isolation of different parts of the structure which is essential for the cuff sensor, and must be modified to meet the needs of the cuff.
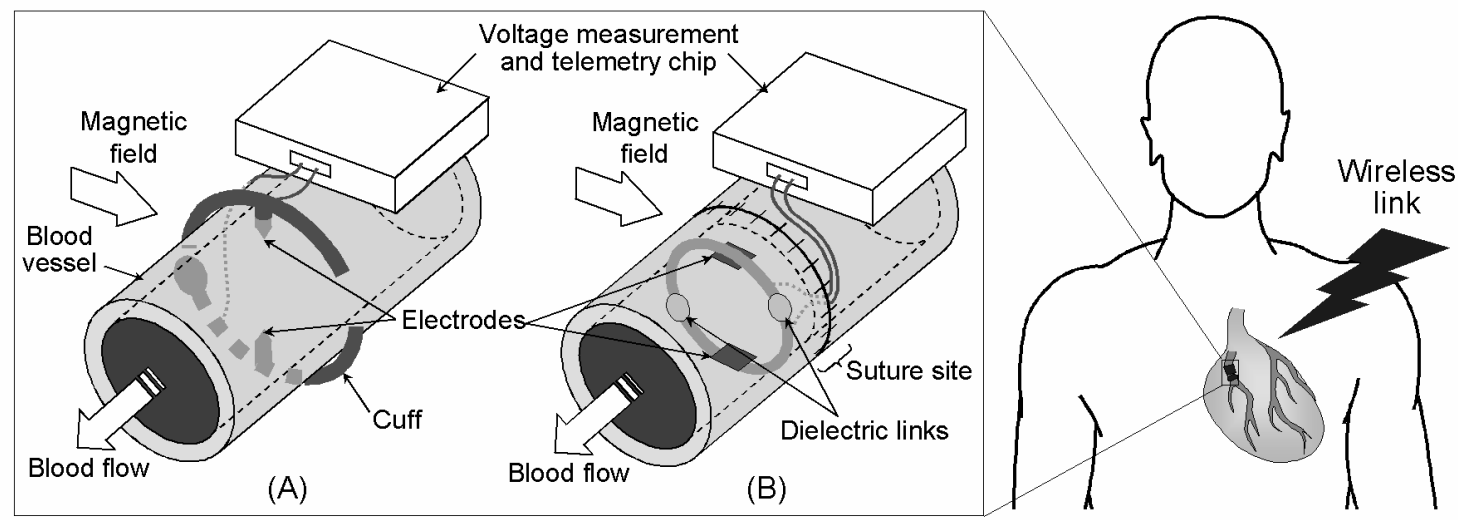

Fig. 1: Conceptual diagram of the device implementation options: (A) A C-shape cuff with penetrating electrodes wrapped around a blood vessel; (B) a ring cuff implanted inside a blood vessel upon vascular surgery. Lead transfer is located at suture sites.

"Corresponding author: 1301 Beal Avenue, Ann Arbor, MI 48109-2122, USA; Tel: +1-734-647-1782; Fax: 763-9324; Email: ktakahat@eecs.umich.edu 
The planar design of the ring cuff has a pair of meander bands comprised of $50 \mu \mathrm{m}$-wide beams, electrode plates, and two dielectric links which mechanically tie the bands but electrically insulate them each other (Fig. 2). This pattern is EDMed in 50 $\mu \mathrm{m}$-thick \#304 stainless steel foil so that two bands are connected to the original foil, maintaining $100-\mathrm{m}$ gaps at the links. Insulating cement is used to bridge the gaps, and then the device is released from the foil (Fig. 3). This is similar to the process flow for a Kelvin probe described in [8]. Lead wires are bonded to the electrodes with conductive adhesive. All surfaces of the device except front-side planes of the electrodes are coated with an insulating layer. (Without this, spatial averaging will reduce the voltage.) The electrode may optionally be coated with an antifouling layer. This feasibility experiment used two-part epoxy and enamel for the cement and the insulation layer respectively.

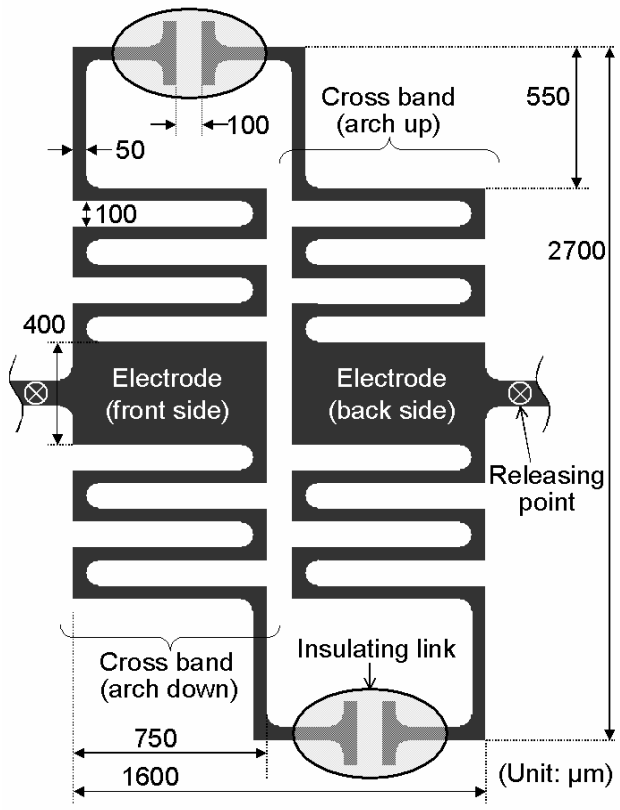

Fig. 2: A layout of the planar cuff structure.
(A)

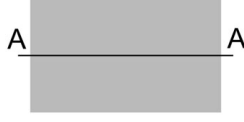

$\mathrm{A}^{\prime}$

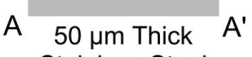
Stainless Steel

(B)

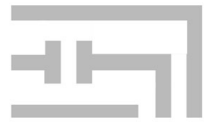

(C)

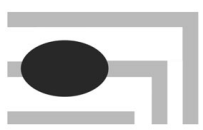

(D)

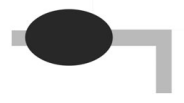

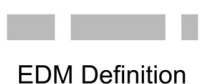
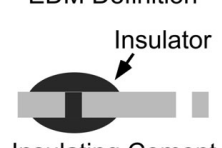

Insulating Cement Fill and Cure

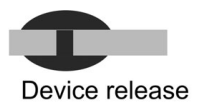

Fig. 3: Fabrication process flow [8].

The planar structure (Fig. 4) is mounted on a deflated balloon, which was a standard angioplasty catheter in this case (Fig. 5), so that one of the bands is located above the balloon whereas the other band is below it (Fig. 6). The device is then deployed near a chronically or potentially diseased location in a blood vessel. Although this device is deployed by an angioplasty balloon, its deployment requires access to both inside and outside of the blood vessel. Thus, it is most appropriate for use during a bypass procedure in which the blood vessels are severed. The lead transfer through the walls of the vessel, as noted previously, can be located at the suture sites. The cuff is expanded by the balloon before the vessel is sutured. Figure 7 shows a device that is expanded inside a silicone tube with $3-\mathrm{mm}$ i.d.. The balloon is inflated up to $7 \mathrm{~atm}$. causing the lumen to expand to $3.5 \mathrm{~mm}$ in diameter. When the balloon is deflated and removed the expanded cuff remains within the tube (Fig. 8). Tests with flow velocities up to $2 \mathrm{~m} / \mathrm{s}$ show that both the structure and its placement are robust and immovable. (Maximum arterial flow is typically $\approx 1.6 \mathrm{~m} / \mathrm{s}$.)

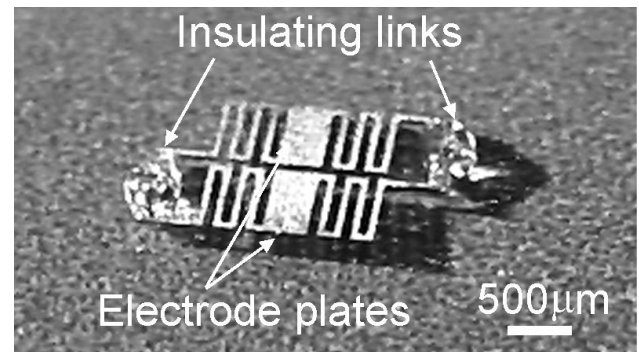

Fig. 4: A stainless steel cuff in the planar form.

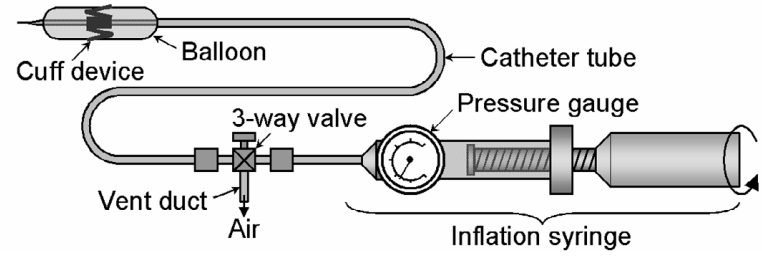

Fig. 5: A set-up for deployment of a cuff device.

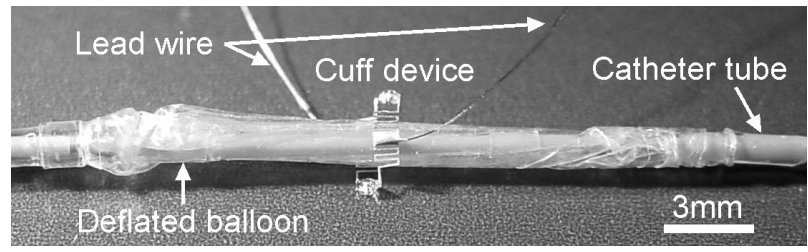

Fig. 6: A planar cuff structure mounted on a standard catheter balloon in the deflated state.

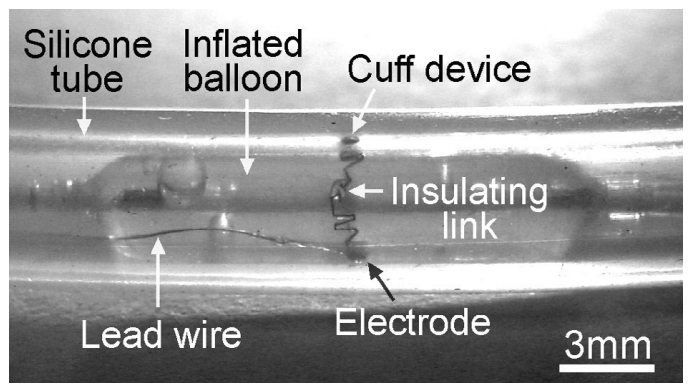

Fig. 7: The stainless steel cuff plastically expanded to a ring shape inside a silicone tube/mock artery by inflation of the balloon.

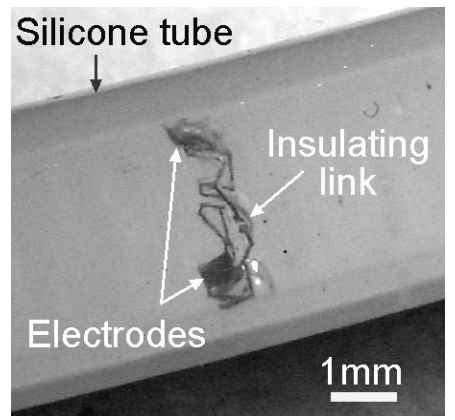

Fig. 8: The cuff remains attached to the on inner walls of the tube by pressure after the balloon is deflated and removed. (The lead wires have been removed.) 


\section{EXPERIMENTAL RESULTS}

Figure 9 shows the measurement set-up: a pump/flowcontroller regulates the flow of $2 \%$ wt. saline and a voltmeter measures voltage between the electrode leads. A permanent magnet with dimensions of $25 \times 25 \times 9 \mathrm{~mm}^{3}$ was used to provide magnetic field in this setup. The field orientation was perpendicular to both flow direction and the voltage sense axis defined by the locations of the two electrodes. The magnetic field was characterized by an InAs Hall sensor (F. W. Bell, FL, model $\mathrm{BH}-205$ ) and measured to be $\approx 0.25 \mathrm{~T}$ at the location of the cuff. The presence of the cuff had no detectable impact on the externally measured magnetic field. To determine the electromagnetic effect, voltage change due to varying flow rate was measured with opposing orientations of the magnetic field as shown in Fig. 10. The voltage change relative to a baseline value, which is associated with polarization and electrochemical effects, is plotted in Fig. 11. The voltage linearly and symmetrically increases or decreases depending on the orientation. The voltage response and sensitivity in this test were 3.1-4.3 V per cm/sec and 50-70 ppm per $\mathrm{cm} / \mathrm{sec}$, respectively.

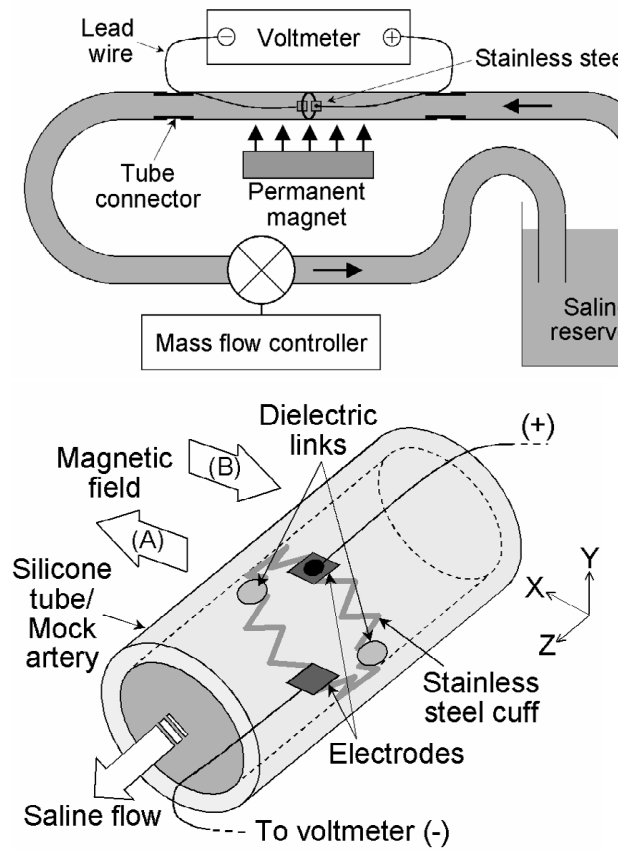

Fig. 10: A closeup of the device in the set-up with different orientations of magnetic field.

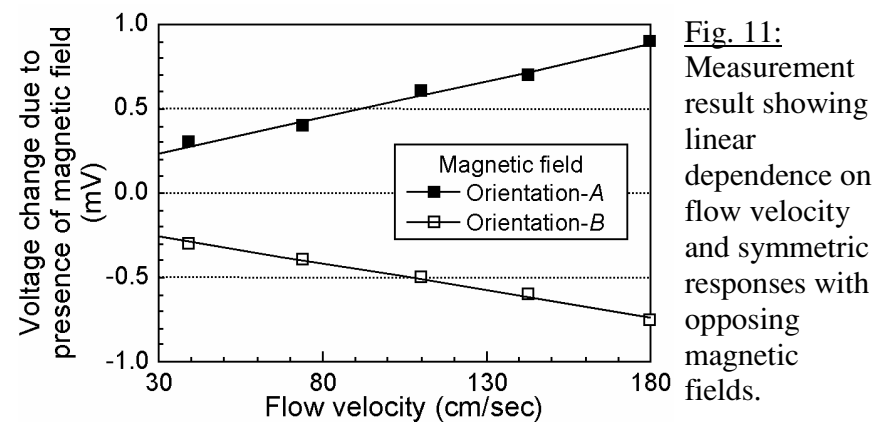

\section{ANALYSIS AND DISCUSSION}

Since the output signal may depend on orientation of the magnetic field, it is important to evaluate the extent of this. Figure 12a illustrates magnetic fields misaligned by $\theta$ in the $X-Z$ plane and $\varphi$ in the misaligned plane perpendicular to the $X-Z$ plane. The cuff is parallel to the $X-Y$ plane which is perpendicular to a flow direction. The electrodes are located at $\varphi=90^{\circ}$ as seen in Fig. 12a.
The induced potential at $\varphi$ on the circular perimeter in the $X-Y$ plane is $\left(E_{\max } / 2\right) \cdot \sin \varphi$ [5]. When the electrodes are located on walls of the flow channel, the modified output voltage that incorporates these angular errors can be expressed as:

$$
\text { E } E_{\max } \cdot \cos \varphi \cdot \cos \theta
$$

Figure $12 \mathrm{~b}$ shows a contour map of voltage normalized by the maximum value i.e. $E_{\max }(\theta=\varphi=0)$ as a function of $\theta$ and $\varphi$ up to 90 , where $E$ becomes zero. For example, when the magnetic field is tilted by 15 for both $\theta$ and $\varphi$, the signal loss is estimated to be $6.7 \%$. As seen in eqn. (2) or the map impact of the angular error is less for smaller angles. This deviation can be minimized by having an external magnet with a proper harness that fits to the geometry of skin surfaces.
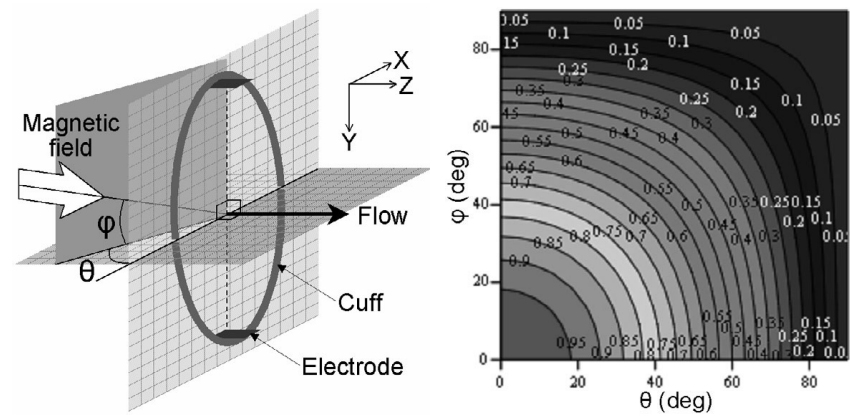

Fig. 12: (a-left) Definition of misaligned angles of magnetic field; and (b-right) a normalized voltage $\left(E / E_{\max }\right)$ map on $\theta-\varphi$ axes.

Using eqn. (1) with $D=3 \mathrm{~mm}$ and $B=0.25 \mathrm{~T}$, the calculated output voltages are plotted with a dotted line (Cal-1) in Fig. 13b. Although at lower flow velocity the prediction matches well with the experimental result (also plotted in Fig. 13b with square dots), it deviates as the flow velocity is increased. Figure 13a shows actual electrode positions observed in the expanded cuff, where they are not actually on a diametrical line but are shifted by approximately 50 in total during the balloon expansion. This nonuniform expansion can lower the output voltage. Two hypothetical cases are evaluated and plotted in Fig. 13b: each of electrodes is equally offset by 25 as shown in Fig. 13a (Cal-2), and one of electrodes is offset by the whole 50 (Cal-3). The plots indicate that the latter case shows closer match to the measurement, although the deviation from the measured values is still not negligible. A potential cause for this mismatch is a loss associated with non-ideal profile of flow velocity. The presence of electric leads which are connected to the electrodes and/or the cuff itself can disturb the flow. The sensitivity to local flow velocity varies across the channel and rapidly increases close to the electrodes [9] Thus, the disturbance near an electrode due to the boundary irregularities can potentially require a correction factor to be introduced into the theoretical estimate, which is otherwise idealized. Lead wires that were attached directly to the electrodes
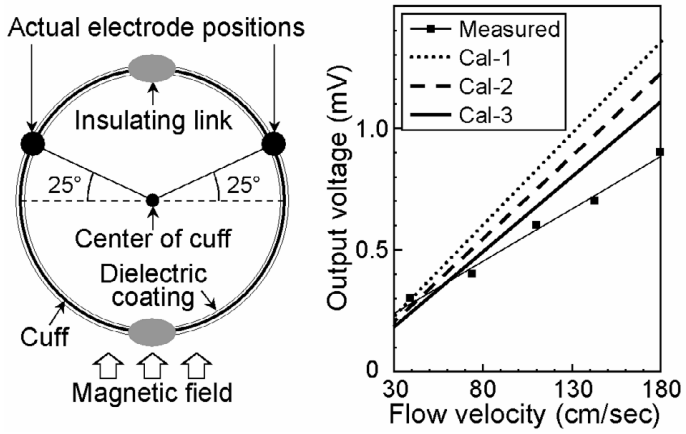

Fig. 13: (a-left) Electrode positions in the cuff structure; (b-right) measured and projected voltages as a function of flow velocity. 
and the use of relatively large electrodes $\left(400 \times 750 \mathrm{~m}^{2}\right)$ in the setup may have also contributed to the reduced response. However, the latter tends to reduce the sensitivity to the electrode position error because of the averaging effect. These issues open an avenue for design optimization of the cuff in the future.

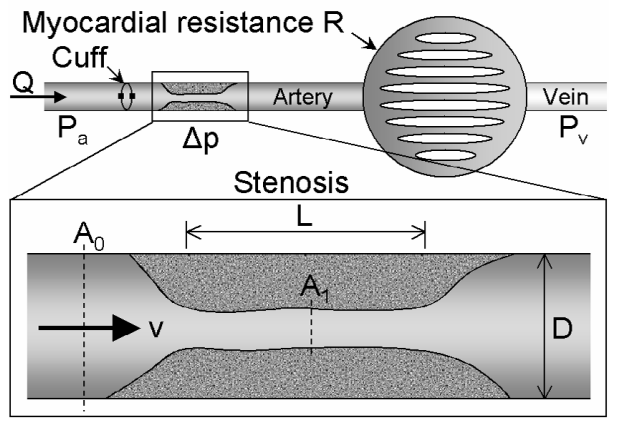

Fig. 14: Simplified model of the vascular system with a stenosis.

The impact of a blockage in a blood vessel (stenosis) on the sensing signal was evaluated using a simplified coronary circulation model with a narrowed artery, as shown in Fig. 14. This model assumes oscillating laminar flow in a rigid-wall vessel and blood as a Newtonian fluid, which is reasonable assumption for arteries with $>\phi 1 \mathrm{~mm}$ [10]. The pressure and flow rate used in this analysis were time-averaged values for one cardiac cycle. The blood flow rate in a normal artery is given by [10]:

$$
Q \frac{P_{a} P_{v} \Delta p}{R_{p}}
$$

where $Q$ is voluminal flow rate, $P_{a}$ and $P_{v}$ are arterial (upstream of the stenosis) and venous pressures respectively, $R$ is myocardial resistance, and $\Delta p$ is the pressure drop across the stenosis. This analysis uses $P_{a}=100 \mathrm{mmHg}, \quad P_{v}=0 \mathrm{mmHg}$, and $R \approx 0.4$ $\mathrm{mmHg} /(\mathrm{ml} / \mathrm{min})$ [11]. The pressure drop can be expressed as [12]:

$$
\Delta p \quad K_{v} \frac{4 Q}{\pi D^{3}} \quad K_{t} \alpha 1^{2} \frac{8 \beta \rho Q^{2}}{\pi^{2} D^{4}}
$$

where $\alpha$ is the ratio of average area of unobstructed artery to minimum cross-sectional area of stenosis i.e. $A_{0} / A_{l}$ in Fig. $14, \beta$ is a function of flow waveform whose average value is about 2.5 [12], $\rho$ is density of blood, and is viscosity of blood. $K_{v}$ and $K_{t}$ are coefficients that depend on stenosis shape and entrance/exit geometrical characteristics, respectively. This analysis assumes a stenosis with circular cross section and constant channel diameter across its length $L$, where the coefficients can be approximated to be $K_{v} \approx 32(L / D) \alpha^{2}$ and $K_{t} \approx 1.5$ for blunt ends, which includes both entrance and exit effects [13]. Using eqns. (3) and (4), the dependence of flow rate $Q$ on the stenosis ratio $\alpha$ can be obtained. Assuming conditions for eqn. (1) and that the cuff is located upstream of the stenosis as shown in Fig. 14, the output voltage can be calculated with the flow rate, or velocity. Figure 15 plots the result as a function of percent stenosis, which means percent reduction of channel area, i.e., (1-1/ $\alpha) 100$, with different diameters of the artery. The plot shows that the growth of the stenosis can be

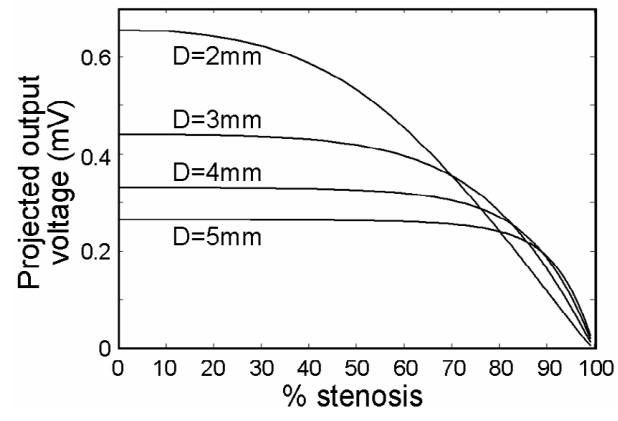

Fig. 15: Projected output voltages vs. percent stenosis with different blood vessel diameters. detected earlier for a narrower blood vessel. The analysis indicates that, for example, when $D=2 \mathrm{~mm}, 10 \%$ change in the output voltage is induced by $39 \%$ stenosis. (Left main coronary artery disease is defined as $>50 \%$ narrowing.)

\section{CONCLUSION}

A micromachined stainless steel cuff for electromagnetic flow sensing in a blood vessel has been investigated. A $50-\mathrm{m}$ thick planar microstructure with overall dimensions of $2.7 \times 1.6 \mathrm{~mm}^{2}$ was designed and fabricated to have a pair of electrodes that were mechanically coupled but electrically isolated by dielectric links. The planar structure was then assembled on a deflated angioplasty balloon, and plastically expanded to ring shape by inflation of the balloon inside a silicone tube. The expanded cuff was lodged on inner walls of the tube by its own pressure and remained immovable in at least $2-\mathrm{m} / \mathrm{s}$ flow. Fluidic tests that used saline and $\mathrm{DC}$ magnetic field of $0.25 \mathrm{~T}$ demonstrated linear response of electromagnetically induced voltage to varied flow speed. The measurement result exhibited reasonable match to theoretical analysis. To circumvent polarization sensitivity and allow amplification of the output signal, the future use of AC magnetic field instead of the DC operation will be continued.

\section{ACKNOWLEDGEMENT}

This research was supported in part by a grant from the National Science Foundation. Travel support has been generously provided by the Transducers Research Foundation and by the DARPA MEMS and DARPA BioFlips programs.

\section{REFERENCES}

[1] A. Bolz, V. Lang, B. Merkely, M. Schaldach, "First Results of an Implantable Sensor for Blood Flow Measurement," IEEE Conf. EMBS, pp. 2341-3, 1997

[2] A. DeHennis, K.D. Wise, "A Fully-Integrated Multi-Site Pressure Sensor for Wireless Arterial Flow Characterization," Solid-State Sensor and Actuator Workshop (Hilton Head), 2004

[3] B.H Brown, R.H. Smallwood, D.C. Barber, P.V. Lawford, D.R. Hose, "Medical Physics and Biomedical Engineering," Institute of Physics Publishing, Bristol and Philadelphia, pp. 608-11, 1999

[4] H.J. Yoon, S.Y. Kim, S.W. Lee, S.S. Yang, "Fabrication of a Micro Electromagnetic Flow Sensor for Micro Flow Rate Measurement," SPIE Symp. Smart Struc. Mat., pp. 264-71, 2000

[5] A. Kolin, "An Alternating Field Induction Flow Meter of High Sensitivity,” Rev. Sci. Instrum., 16(5), pp.109-16, 1945

[6] K. Takahata, Y.B. Gianchandani, "Batch Mode Micro-ElectroDischarge Machining," IEEE J. MEMS, 11(2), pp.102-10, 2002

[7] K. Takahata, Y.B. Gianchandani, "Coronary Artery Stents Microfabricated from Planar Metal Foil: Design, Fabrication, and Mechanical Testing," IEEE Conf. MEMS, pp. 462-5, 2003

[8] L.L. Chu, K. Takahata, P. Selvaganapathy, J.L. Shohet, Y.B. Gianchandani, "A Micromachined Kelvin Probe for Surface Potential Measurements in Microfluidic Channels and Solid-State Applications," IEEE Conf. Solid-State Sensor, Actuators, and Microsystems (Transducers), pp. 384-7, 2003

[9] J.A. Shercliff, "The Theory of Electromagnetic FlowMeasurement," Cambridge University Press, 1962

[10] D.F. Young, "Fluid Mechanics of Arterial Stenosis," $J$. Biomech. Eng., 101, pp. 157-75, 1979

[11] P. Segers, G. Fostier, J. Neckebroeck, P. Verdonck, “Assesing Coronary Artery Stenosis Severity: In Vitro Validation of the Concept of Fractional Flow Reserve," Catheter. Cardiovasc. Interv., 46, pp. 375-9, 1999

[12] A.C. Roth, D.F. Young, N.R. Cholvin, "Effect of Collateral and Peripheral Resistance on Blood Flow through Arterial Stenosis," J. Biomech., 9, pp. 367-75, 1976

[13] D.F. Young, "Some Factors Affecting Pressure-Flow Relationships for Arterial Stenosis," ASME Conf. Appl. Mech. Bioeng. Flu. Eng., pp. 87-90, 1983 